\title{
SOIL CHEMICAL PROPERTIES RELATED TO ACIDITY UNDER SUCCESSIVE PIG SLURRY APPLICATIONS ${ }^{(1)}$
}

\author{
Cledimar Rogério Lourenzi ${ }^{(2)}$, Carlos Alberto Ceretta ${ }^{(3)}$, Leandro \\ Souza da Silva ${ }^{(4)}$, Gustavo Trentin ${ }^{(5)}$, Eduardo Girotto ${ }^{(6)}$, Felipe \\ Lorensini $^{(7)}$, Tadeu Luis Tiecher ${ }^{(8)} \&$ Gustavo Brunetto ${ }^{(9)}$
}

\begin{abstract}
SUMMARY
Pig slurry application as soil manure can alter the chemical properties of the soil and affect its acidity, modifying the environment for crop growth and development. The objective of this study was to evaluate the chemical properties related to soil acidity subjected to successive applications of pig slurry. The experiment was conducted in May 2000, in an experimental area of the Federal University of Santa Maria (UFSM) under no-tillage and lasted until January 2008. Nineteen surface applications of $0,20,40$, and $80 \mathrm{~m}^{3} \mathrm{ha}^{-1}$ of pig slurry were performed, during a period of 100 months and the soil sampled in the end (layers $0-2,2-4,4-6,6-8,8-10,10-12,12-14,14-16,16-18,18-20,20-25,25-30,30-35,35-40$, 40-50 and 50-60 cm). The application of pig slurry increased soil $\mathrm{pH}$ values, an effect that could reach the depth of $8 \mathrm{~cm}$ without affecting the potential acidity values. The applications also resulted in accumulation of $\mathrm{Ca}$ and $\mathrm{Mg}$ exchangeable levels in the surface layers, increasing base saturation and reducing $\mathrm{Al}$ saturation.
\end{abstract}

(1) Part of the master's thesis of the first author for the Graduate Study Program in Soil Science (PPGCS) of the Universidade Federal de Santa Maria (UFSM). Received for publication in April 23, 2010 and approved in July 7, 2011.

${ }^{(2)}$ Agronomist, Master in Soil Science, Doctoral Candidate in the PPGCS at the UFSM, Rural Sciences Center - CCR. Caixa Postal 221, CEP 97105-900 Santa Maria (RS), Brazil. Doctoral studies scholarship holder from the CAPES. E-mail: lourenzicr@gmail.com

(3) Agronomist, Doctor in Soil Science, Professor of the Soil Department at the UFSM, CCR. Caixa Postal 221, CEP 97105-900 Santa Maria (RS), Brazil. Scholarship holder of Productivity in Research from the CNPq. E-mail: carlosceretta@ufsm.br

${ }^{(4)}$ Agronomist, Doctor in Soil Science, Professor of the Soil Department at the UFSM, CCR. Caixa Postal 221, CEP 97105-900 Santa Maria (RS), Brazil. E-mail: leandro@smail.ufsm.br

(5) Agronomist, Doctor in Agronomy, Research at the Brazilian Agricultural Research Corporation South, Bagé (RS). E-mail: gustavotrentin@yahoo.com.br

(6) Agronomist, Doctor in Soil Science, Professor of the Instituto Federal de Educação, Ciência e Tecnologia do Rio Grande do Sul, Ibirubá (RS). E-mail: eduardogirotto@hotmail.com

(7) Agronomist, Master in Soil Science, Doctoral candidate in the PPGCS at the UFSM. Doctoral studies scholarship holder from the CNPq. E-mail: felipe.lorensini@hotmail.com

(8) Student of Agronomy at the UFSM. Email: tadeu.t@hotmail.com

(9) Agronomist, Doctor in Soil Science, Professor of the Soil Department at the Federal University of Santa Catarina (UFSC), Center of agricultural science, Rural engineering department, Admar Gonzaga highway, 1346, CEP 88034-000 Florianópolis (SC). E-mail: brunetto.gustavo@gmail.com 
Long-term applications induced an increase in organic matter in the deeper layers. However, the effect of this residue on the potential CEC was less significant and restricted to the surface layers.

Index terms: water $\mathrm{pH}$, base saturation, $\mathrm{Al}$ saturation, soil organic matter, organic waste.

\title{
RESUMO: ATRIBUTOS QUIIMICOS RELACIONADOS A ACIDEZ EM SOLO COM SUCESSIVAS APLICAÇÕES DE DEJETO LÍQUIDO DE SUÍNOS
}

\begin{abstract}
A aplicação de dejetos como adubo ao solo pode alterar os atributos químicos deste e afetar a sua acidez, modificando o ambiente para o crescimento e desenvolvimento das culturas. O objetivo deste trabalho foi avaliar a alteração de atributos químicos relacionados à acidez de um solo submetido a aplicações sucessivas de dejeto líquido de suínos. O experimento foi instalado em maio de 2000, em área experimental sob plantio direto pertencente à Universidade Federal de Santa Maria (UFSM) e conduzido até janeiro de 2008. Foram realizadas 19 aplicações superficiais de 0, 20, 40 e $80 \mathrm{~m}^{3} \mathrm{ha}^{-1}$ de dejeto líquido de suínos, durante 100 meses. Ao final, foi coletado solo nas camadas de 0-2, 2-4, 4-6, 6-8, 8-10, 10-12, 12-14, 14-16, 16 18, 18-20, 20-25, 25-30, 30-35, 35-40, 40-50 e 50-60 cm. A aplicação de dejeto líquido de suínos aumentou os valores de $\mathrm{pH}$ do solo, podendo o incremento atingir a profundidade de $8 \mathrm{~cm}$, sem alteração nos valores de acidez potencial. Também promoveu acúmulo dos teores trocáveis de Ca e Mg nas camadas superficiais do solo, aumentando os valores de saturação por bases e diminuindo a saturação por Al. As aplicações ao longo do tempo possibilitaram aumento no teor de MO em profundidade. Entretanto, o efeito desses dejetos sobre a CTC potencial foi menos significativo e restrito à camada superficial.
\end{abstract}

Termos para indexação: $p H$ em água, saturação por bases, saturação por alumínio, matéria orgânica do solo, resíduos orgânicos.

\section{INTRODUCTION}

Pig farming in confined systems has increased in the last years in the Southern region of Brazil, especially in the states of Rio Grande do Sul (RS) and Santa Catarina (SC). Consequently, the production of pig slurry also increased. The residue has been applied in cultivated areas with annual crops and pastures as organic nutrient source. However, in general, high rates of manure applied frequently can (over time) result in the accumulation of nutrients in the soil, surpassing crop uptake capacity and requirements (Basso et al., 2005; Berwanger et al., 2008; McDowell et al., 2011). Organic waste application at high rates, along with continuous cultivation without soil tillage can cause, in the long term, alterations in soil chemical properties related to acidity (Ceretta et al., 2003). Therefore, the response to the question whether liming is required and at which rate, can change in soils after successive applications of pig slurry.

In acid soils, cattle manure application can cause increases in soil $\mathrm{pH}$ values (Whalen et al., 2000). According to these authors, the reason is the presence of $\mathrm{CaCO}_{3}$ in the animal diet, which is not totally absorbed by the animal, being partly excreted. Additionally, the authors stated that the effect of manure on soil $\mathrm{pH}$ depends on the manure source and soil properties. But according to Eghball (2002), the application of organic waste to soil can alter the soil $\mathrm{pH}$ values. This alteration depends on the initial soil $\mathrm{pH}$ at the moment of application. The organic compounds in pig slurry are also very important in the chemical reaction that occurs in the soil, especially in surface layers, for reducing the toxicity of $\mathrm{Al}$ and heavy metals such as $\mathrm{Cu}$ and $\mathrm{Zn}$, found at high concentrations in pig slurry (Girotto, 2007; Brown et al., 2008). The complexation of exchangeable $\mathrm{Al}$ and consequent reduction in $\mathrm{Al}$ saturation was observed by Ceretta et al. (2003) in a study using applications of 0,20 and $40 \mathrm{~m}^{3} \mathrm{ha}^{-1}$ of pig slurry to natural pasture under a Hapludult. As reported by the authors, this effect is mainly due to the action of the fulvic and humic acid fractions of the organic matter, which are very reactive and increasingly significant in soils with high $\mathrm{C}$ addition.

The nutrient concentration in manure is unbalanced in relation to the crop requirements (Seganfredo, 1999; Berwanger et al., 2008). This often implies in the addition of nutrient quantities over plant 
requirements. Among these nutrients are $\mathrm{Ca}$ and $\mathrm{Mg}$, which accumulate in the soil and affect the chemical properties related to acidity (such as base saturation), when applied in great quantities. Soil organic matter also undergoes alterations in pig slurry-treated soils. Apart from the organic compounds found in the manure, the higher production of dry matter by the manure-treated crops contribute to a greater accumulation of organic matter. Adeli et al. (2008), who studied the alterations of chemical properties in three soil types with pig slurry application, observed an increase in the organic matter levels to a depth of $15 \mathrm{~cm}$ in both soils. One of the main functions of the mineralized organic matter in the soil is to be a nutrient source for plants, particularly of N, S and P. Furthermore, the surface electric charges of the organic matter affect the chemical properties, e.g, the cation exchange capacity of the soil and consequently, $\mathrm{Al}$ and base saturation.

The objective of this study was to evaluate the alterations of the chemical properties related to soil acidity in a soil under successive applications of pig slurry in a no-tillage system.

\section{MATERIAL AND METHODS}

The experiment was conducted in the experimental area of the Agricultural Engineering Department at the Federal University of Santa Maria (UFSM), in Santa Maria - RS. The soil used was Hapludult (Soil Survey Staff, 1999), with sandy loam surface texture on a $4 \%$ slope. The area was maintained for eight years under a no-tillage system until March 2000. The following physical-chemical properties were determined in the $0-10 \mathrm{~cm}$ soil layer: clay $170 \mathrm{~g} \mathrm{~kg}^{-1}$, silt $300 \mathrm{~g} \mathrm{~kg}^{-1}$ and sand $530 \mathrm{~g} \mathrm{~kg}^{-1}$, by the pipette method (USDA, 1972); $\mathrm{pH}-\mathrm{H}_{2} \mathrm{O} 4.7, \mathrm{Ca} 2.7 \mathrm{cmol}_{\mathrm{c}} \mathrm{dm}^{-3}$ and $\mathrm{Mg} 1.1 \mathrm{cmol}_{\mathrm{c}} \mathrm{m}^{-3}$, determined as proposed by Tedesco et al. (1995); $\mathrm{H}+\mathrm{Al} 5.6 \mathrm{cmol}_{\mathrm{c}} \mathrm{dm}^{-3}$, determined by the extraction of calcium acetate of $0.5 \mathrm{~mol} \mathrm{~L}^{-1}$ at pH 7.0 and subsequent titration with $\mathrm{NaOH}$ $0.025 \mathrm{~mol} \mathrm{~L}^{-1} ; \mathrm{Al}$ saturation of $17 \%$, base saturation of $42 \%$ and potential cation exchange capacity $\left(\mathrm{CEC}_{\mathrm{pH}}\right) 9.6 \mathrm{cmol}_{\mathrm{c}} \mathrm{dm}^{-3}$, calculated in agreement with the equations proposed by the Comissão de Fertilidade do Solo - RS/SC (CFRS/SC, 1995); organic matter $16 \mathrm{~g} \mathrm{dm}^{-3}$, calculated from the total organic C (TOC) levels obtained according to the method proposed by Embrapa (1997).

In May 2000, pig slurry rates of 0, 20,40, and $80 \mathrm{~m}^{3} \mathrm{ha}^{-1}$ were applied. These rates were applied 19 times during the experiment. The manure came from dunghills of farms in the Central Depression region of RS (Table 1).

The pig slurry was scattered on the soil surface without soil incorporation, the day before sowing of each species, as the only nutrient source for the crops. The plots $(4 \times 3 \mathrm{~m})$ were arranged in a randomized block design with three replications. During the experiment, the following crop succession/sequence was evaluated: black oat (Avena strigosa) / maize (Zea mays L.)/wild radish (Raphanus sativus L.), during the growing season of 00/01 and 01/02 (Ceretta et al., 2005); black oat/maize (Pennisetum americanum L.)/ black bean (Phaseolus vulgaris L.) in 02/03; black oat/common vetch (Vicia sativa L.)/maize in 03/04 and 04/05; black oat/black bean/Sunnhemp (Crotalaria juncea L.) in 05/06; black oat/maize/black oat in 06/ 07.

In January 2008, 100 months after the beginning of the experiment, the soil was sampled in the layers $0-2,2-4,4-6,6-8,8-10,10-12,12-14,14-16,16-$ $18,18-20,20-25,25-30,30-35,35-40,40-50$ and $50-60 \mathrm{~cm}$. The soil was collected with a shovel, airdried and crushed manually using a heavy roll. Then the soil was sieved through $2 \mathrm{~mm}$ mesh and stored. Water $\mathrm{pH}$ (1:1) and exchangeable levels of $\mathrm{Al}, \mathrm{Ca}$ and Mg were determined according to the method proposed by Tedesco et al. (1995). From these data, the potential cation exchange capacity $\left(\mathrm{CEC}_{\mathrm{pH} 7}\right), \mathrm{Al}$ saturation $(\mathrm{m})$, base saturation $(\mathrm{V})$ and potential acidity $(\mathrm{H}+\mathrm{Al})$ were calculated, by the equations proposed by CQFSRS/SC (2004). Besides, total organic carbon (TOC) levels were determined, according to the method described by Embrapa (1997), with adaptations of the heating intervals of the block digester, as described by Girotto (2007). To calculate the soil organic matter (SOM) value, the total organic C levels were multiplied by 1.724 (Van Bemmelen factor), assuming that $58 \%$ of the organic matter of the soils consists of organic carbon.

The variable factors evaluated were the applied residue rates and the soil horizons. The interaction between the factors was tested by variance analysis at $5 \%$. When the qualitative results between soil layers were significant, the average values were compared by the Scott-Knott test at $5 \%$. For the quantitative data, when significant differences were shown, equations were adjusted and subjected to variance analysis, test $\mathrm{F}$, test $\mathrm{T}$ and to the coefficient of determination at $5 \%$.

\section{RESULTS AND DISCUSSION}

The variance analysis demonstrated that the effects of manure rates and soil layers on the variables water $\mathrm{pH}, \mathrm{Al}$ saturation, base saturation, exchangeable $\mathrm{Ca}$ and $\mathrm{Mg}, \mathrm{SOM}$ and potential CEC were statistically significant. With regard to potential acidity, only the effect of soil layers was significant. The interaction between the factors waste rate $\mathrm{x}$ soil layer was not significant at $5 \%$ for water $\mathrm{pH}$, potential acidity, $\mathrm{Al}$ saturation, base saturation, exchangeable $\mathrm{Ca}$ and $\mathrm{Mg}$, 
Table 1. Pig slurry characteristics and nutrient amount applied before the implantation of each crop during the experimental period

Pig slurry characteristic

\begin{tabular}{|c|c|c|c|c|}
\hline Dry matter (\%) & \multicolumn{4}{|c|}{$\begin{array}{c}\text { Black Oat } \\
1.9\end{array}$} \\
\hline $\begin{array}{l}\text { Total Potassium } \\
\text { Total Calcium }^{(1)} \\
\text { Total Magnesium }^{(1)}\end{array}$ & $\begin{array}{l}\% \\
1.2 \\
3.4 \\
1.3\end{array}$ & $\begin{array}{r}20 \\
4.5 \\
12.6 \\
4.9\end{array}$ & $\begin{array}{r}40 \\
8.9 \\
25.2 \\
9.7\end{array}$ & $\begin{array}{r}80 \\
17.8 \\
50.4 \\
19.4\end{array}$ \\
\hline Dry matter (\%) & & Appli & $\begin{array}{l}\text { k Oat } \\
1 \\
\text { tion - }\end{array}$ & $\mathrm{g} \mathrm{ha}^{-1}$ \\
\hline $\begin{array}{l}\text { Total Potassium } \\
\text { Total Calcium }^{(1)} \\
\text { Total Magnesium }^{(1)}\end{array}$ & $\begin{array}{l}\% \\
1.30 \\
2.8 \\
1.3\end{array}$ & $\begin{array}{l}20 \\
2.9 \\
6.2 \\
2.9\end{array}$ & $\begin{array}{r}40 \\
5.8 \\
12.4 \\
5.8\end{array}$ & $\begin{array}{r}80 \\
11.6 \\
24.8 \\
13.2\end{array}$ \\
\hline Dry matter (\%) & \multicolumn{4}{|c|}{$\begin{array}{l}\quad 0.47 \\
\text { Application - } \mathrm{kg} \mathrm{ha}^{-1}\end{array}$} \\
\hline $\begin{array}{l}\text { Total Potassium }^{(1)} \\
\text { Total Calcium }^{(1)} \\
\text { Total Magnesium }^{(1)}\end{array}$ & $\begin{array}{l}\% \\
0.41 \\
4.40 \\
0.96\end{array}$ & $\begin{array}{l}20 \\
0.4 \\
4.2 \\
0.9\end{array}$ & $\begin{array}{l}40 \\
0.8 \\
8.4 \\
1.8\end{array}$ & $\begin{array}{r}80 \\
1.6 \\
16.8 \\
3.6\end{array}$ \\
\hline
\end{tabular}

Dry matter (\%)

Black Oat + Common vetch

\begin{tabular}{|c|c|c|c|}
\hline & & \multicolumn{2}{|c|}{ Application $-\mathrm{kg} \mathrm{ha}^{-1}$} \\
\hline & $\%$ & 20 & 40 \\
\hline Total Potassium ${ }^{(1)}$ & 2.90 & 13.7 & 27.5 \\
\hline Total Calcium ${ }^{(1)}$ & 1.91 & 9.0 & 18.0 \\
\hline Total Magnesium ${ }^{(1)}$ & 3.27 & 15.5 & 31.0 \\
\hline \multirow{4}{*}{ Dry matter (\%) } & \multicolumn{3}{|c|}{ Black Oat + Common vetch } \\
\hline & \multicolumn{3}{|c|}{1.0} \\
\hline & \multicolumn{3}{|c|}{ Application $-\mathrm{kg} \mathrm{ha}^{-1}$} \\
\hline & $\%$ & 20 & 40 \\
\hline Total Potassium ${ }^{(1)}$ & 10.13 & 20.3 & 40.5 \\
\hline Total Calcium ${ }^{(1)}$ & 1.84 & 3.7 & 7.4 \\
\hline Total Magnesium ${ }^{(1)}$ & 3.37 & 6.7 & 13.4 \\
\hline
\end{tabular}

Dry matter $(\%)$

$\begin{array}{lcrrr} & \% & 20 & 40 & 80 \\ \text { Total Potassium }^{(1)} & 0.91 & 12.4 & 24.8 & 49.6 \\ \text { Total Calcium }^{(1)} & 1.32 & 18.0 & 36.0 & 72.0 \\ \text { Total Magnesium }^{(1)} & 2.35 & 32.0 & 64.0 & 128.0\end{array}$

Dry matter (\%)

Black oat

$$
9.94
$$

Application - $\mathrm{kg} \mathrm{ha}^{-1}$

Total Potassium
Total $^{(1)}$

Total Magnesium ${ }^{(1)}$

\begin{tabular}{cccc}
\multicolumn{4}{c}{ Application $-\mathrm{kg}$ ha } \\
$\%$ & 20 & \multicolumn{1}{c}{40} & \multicolumn{1}{c}{80} \\
1.85 & 36.8 & 73.6 & 147.2 \\
1.26 & 25.0 & 50 & 100 \\
2.35 & 46.7 & 93.4 & 186.8
\end{tabular}

$\begin{array}{cccc}\text { First agricultural year }(00 / 01) \\ \text { Maize } \\ 9.9 \\ \% \\ \% & 20 & 40 & 80 \\ 4.6 & 91.4 & 183 & 365 \\ 1.8 & 35.8 & 71.6 & 143.2 \\ 1.4 & 27.8 & 55.6 & 111.2\end{array}$

Second agricultural year (01/02)

Maize

$$
3.7
$$

\begin{tabular}{lrcc}
\multicolumn{4}{c}{ Application $-\mathrm{kg} \mathrm{ha}^{-1}$} \\
$\%$ & 20 & 40 & 80 \\
2.10 & 15.5 & 31 & 62 \\
2.9 & 21.5 & 42.9 & 85.8 \\
1.3 & 9.6 & 19.2 & 38.4
\end{tabular}

Third agricultural year $(02 / 03)$

$$
\text { Maize }
$$

$$
6.68
$$

$$
\text { Application - } \mathrm{kg} \mathrm{ha}^{-1}
$$

$$
\begin{array}{llll}
\% & 20 & 40 & 80
\end{array}
$$

$\begin{array}{llll}0.72 & 9.6 & 19.2 & 38.5\end{array}$

$\begin{array}{llll}1.27 & 17.0 & 34.0 & 68.0\end{array}$

$\begin{array}{llll}1.19 & 15.9 & 31.8 & 63.6\end{array}$

Fourth agricultural year (03/04)

Maize

2.07

\begin{tabular}{rcrrr} 
& \multicolumn{4}{c}{ Application $-\mathrm{kg} \mathrm{ha}^{-1}$} \\
80 & $\%$ & 20 & 40 & 80 \\
55.0 & 4.58 & 19.0 & 38.0 & 76.0 \\
36.0 & 1.40 & 5.8 & 11.6 & 23.2 \\
62.0 & 2.67 & 11.0 & 22.0 & 44.0
\end{tabular}

Fifth agricultural year (04/05)

Maize

$$
7.2
$$

Application - $\mathrm{kg} \mathrm{ha}^{-1}$

$\begin{array}{rcrrr}80 & \% & 20 & 40 & 80 \\ 81.0 & 1.27 & 18.3 & 36.6 & 73.2 \\ 14.8 & 3.50 & 50.4 & 100.8 & 201.6 \\ 26.8 & 2.94 & 42.3 & 84.6 & 169.2\end{array}$

Sixth agricultural year (05/06)

Black Bean

$$
8.9
$$

Sunnhemp

$$
12.14
$$

\begin{tabular}{crrr}
\multicolumn{4}{c}{ Application $-\mathrm{kg} \mathrm{ha}^{-1}$} \\
$\%$ & 20 & 40 & 80 \\
2.44 & 33.2 & 66.4 & 132.8 \\
1.45 & 25.8 & 51.6 & 103.2 \\
1.50 & 26.7 & 53.4 & 106.8
\end{tabular}

Application - $\mathrm{kg} \mathrm{ha}^{-1}$

$\begin{array}{cccc}\% & 20 & 40 & 80 \\ 1.01 & 24.5 & 49.0 & 98.0 \\ 1.31 & 31.8 & 63.6 & 127.2 \\ 2.00 & 48.6 & 97.2 & 194.4\end{array}$

Seventh agricultural year (06/07)

$$
\text { Maize }
$$$$
1.91
$$

$$
\text { Application - kg ha }{ }^{-1}
$$

$\begin{array}{crrr}\% & 20 & 40 & 80 \\ 3.56 & 13.6 & 27.2 & 54.4 \\ 1.13 & 4.4 & 8.8 & 17.6 \\ 2.51 & 9.6 & 19.2 & 38.4\end{array}$

Black oat 3.52

Application - kg ha ${ }^{-1}$

$\begin{array}{ccrc}\% & 20 & 40 & 80 \\ 3.58 & 25.2 & 50.4 & 100.8 \\ 3.98 & 28.0 & 56.0 & 112.0 \\ 7.39 & 52.0 & 104.0 & 208.0\end{array}$

(1) Analysis in dry base. ${ }^{(2)}$ Addition of $\mathrm{K}$ with the manure, by its relation to base saturation in soil were shown. 
and potential CEC. Interaction occurred only between the factors in SOM.

The successive pig slurry applications increased the values of water $\mathrm{pH}$ in the soils to a depth of $8 \mathrm{~cm}$ (Table 2). At the $80 \mathrm{~m}^{3} \mathrm{ha}^{-1}$ rate, the increments were present to a depth of $25 \mathrm{~cm}$. However, these results may be related to higher $\mathrm{pH}$ values observed in 10 $20 \mathrm{~cm}$ layer in treatment without waste application. The existing explanations in the literature for this phenomenon differ largely. According to Hue \& Licudine (1999), the addition of organic waste to acid soils can induce higher water $\mathrm{pH}$ values in soil, by $\mathrm{H}^{+}$ ion adsorption by decaying crop residue, which is part of the non-humic fraction of SOM. On the other hand, Chantigny et al. (2004) reported that the higher soil $\mathrm{pH}$ may have been caused by the alkaline properties of pig slurry and also by the dissociation of carbonates from the residue. Paul \& Beauchamp (1989) also emphasize that the fast oxidation of the volatile fatty acids found in pig slurry can cause a rise in $\mathrm{pH}$ values, in agreement with Sorensen (1998), who reported an increase in soil $\mathrm{pH}$ values in a study on the application of volatile fatty acids in incubation experiments. The relevance of these results can be shown by the fact that manure application resulted in a $\mathrm{pH}$ increase from 4.70 in the $0-10 \mathrm{~cm}$ soil layer before the experiment, considered very low according to CQFSRS/SC (2004), to values of up to 5.33, in the same soil layer, by an application of $80 \mathrm{~m}^{3} \mathrm{ha}^{-1}$. In addition, soil sampling in no-tillage for the states of $\mathrm{RS}$ and $\mathrm{SC}$ is recommended in the $0-10 \mathrm{~cm}$ layer. The fact that there were increments in $\mathrm{pH}$ down to $8 \mathrm{~cm}$ could mean important reflexes in the decision making about the need for soil acidity correction, once the referential target $\mathrm{pH}$ in this soil management system is 5.5 in these states.

Nevertheless, results from other studies using pig slurry show that in soil with initial $\mathrm{pH}$ values between

Table 2. Values of water $\mathrm{pH}$ and $\mathrm{H}+\mathrm{Al}$ in soil layers after 19 pig slurry applications at different rates

\begin{tabular}{|c|c|c|c|c|c|c|}
\hline \multirow{2}{*}{ Layer } & \multicolumn{4}{|c|}{ Rate of pig slurry $\left(\mathrm{m}^{3} \mathrm{ha}^{-1}\right)$} & \multirow{2}{*}{$\begin{array}{c}\text { Regression } \\
\text { Equation }\end{array}$} & \multirow{2}{*}{$\mathbf{R}^{2}$} \\
\hline & 0 & 20 & 40 & 80 & & \\
\hline $\mathrm{cm}$ & \multicolumn{4}{|c|}{$\mathrm{pH}$ in water } & & \\
\hline $0-2$ & $5.05 \mathrm{c}^{(1)}$ & $5.37 \mathrm{a}^{(1)}$ & $5.37 \mathrm{a}^{(1)}$ & $5.43 \mathrm{a}^{(1)}$ & $\hat{y}=5.169+0.004 x$ & 0.62 * \\
\hline $2-4$ & $4.95 \mathrm{c}$ & $5.14 \mathrm{~b}$ & $5.18 \mathrm{~b}$ & $5.31 \mathrm{a}$ & $\hat{\mathrm{y}}=4.998+0.004 \mathrm{x}$ & 0.90 * \\
\hline $4-6$ & $4.94 \mathrm{c}$ & $5.03 \mathrm{~b}$ & $5.16 \mathrm{~b}$ & $5.29 \mathrm{a}$ & $\hat{\mathrm{y}}=4.949+0.004 \mathrm{x}$ & 0.98 * \\
\hline $6-8$ & $5.01 \mathrm{c}$ & $5.10 \mathrm{~b}$ & $5.16 \mathrm{~b}$ & $5.32 \mathrm{a}$ & $\hat{\mathrm{y}}=5.014+0.004 \mathrm{x}$ & 0.99 * \\
\hline $8-10$ & $5.10 \mathrm{~b}$ & $5.18 \mathrm{~b}$ & $5.15 \mathrm{~b}$ & $5.31 \mathrm{a}$ & $\mathrm{ns}$ & \\
\hline $10-12$ & $5.13 \mathrm{~b}$ & $5.21 \mathrm{~b}$ & $5.19 \mathrm{~b}$ & $5.28 \mathrm{a}$ & $\mathrm{ns}$ & \\
\hline $12-14$ & $5.17 \mathrm{a}$ & $5.26 \mathrm{a}$ & $5.20 \mathrm{~b}$ & $5.39 \mathrm{a}$ & $\mathrm{ns}$ & \\
\hline $14-16$ & $5.23 \mathrm{a}$ & $5.29 \mathrm{a}$ & $5.25 \mathrm{a}$ & $5.37 \mathrm{a}$ & ns & \\
\hline $16-18$ & $5.20 \mathrm{a}$ & $5.32 \mathrm{a}$ & $5.30 \mathrm{a}$ & $5.38 \mathrm{a}$ & ns & \\
\hline $18-20$ & $5.24 \mathrm{a}$ & $5.32 \mathrm{a}$ & $5.32 \mathrm{a}$ & $5.34 \mathrm{a}$ & $\mathrm{ns}$ & \\
\hline $20-25$ & $5.13 \mathrm{~b}$ & $5.31 \mathrm{a}$ & $5.30 \mathrm{a}$ & $5.19 \mathrm{a}$ & $\mathrm{ns}$ & \\
\hline $25-30$ & $5.15 \mathrm{~b}$ & $5.18 \mathrm{~b}$ & $5.24 \mathrm{a}$ & $5.12 \mathrm{~b}$ & ns & \\
\hline $30-35$ & $5.11 \mathrm{~b}$ & $5.14 \mathrm{~b}$ & $5.15 \mathrm{~b}$ & $5.01 \mathrm{~b}$ & $\mathrm{~ns}$ & \\
\hline $35-40$ & $5.10 \mathrm{~b}$ & $5.09 \mathrm{~b}$ & $5.11 \mathrm{~b}$ & $4.97 \mathrm{~b}$ & $\mathrm{~ns}$ & \\
\hline $40-50$ & $5.07 \mathrm{~b}$ & $5.08 \mathrm{~b}$ & $5.05 \mathrm{c}$ & $4.94 \mathrm{~b}$ & ns & \\
\hline $50-60$ & $5.10 \mathrm{~b}$ & $5.10 \mathrm{~b}$ & $5.02 \mathrm{c}$ & $4.92 \mathrm{~b}$ & $\mathrm{~ns}$ & \\
\hline \multirow[t]{2}{*}{ CV (\%) } & 1.28 & 2.36 & 1.36 & 1.66 & & \\
\hline & \multicolumn{4}{|c|}{$\mathrm{H}+\mathrm{Al}\left(\mathrm{cmol}_{\mathrm{c}} \mathrm{dm}^{-3}\right)$} & & \\
\hline $0-2$ & $5.0 \mathrm{~b}^{(1)}$ & $4.0 \mathrm{~b}^{(1)}$ & $3.9 b^{(1)}$ & $3.8 b^{(1)}$ & $\mathrm{ns}$ & \\
\hline $2-4$ & $5.4 \mathrm{~b}$ & $4.8 \mathrm{~b}$ & $4.4 \mathrm{~b}$ & $4.3 \mathrm{~b}$ & $\mathrm{~ns}$ & \\
\hline $4-6$ & $5.7 \mathrm{~b}$ & $5.2 \mathrm{~b}$ & $4.7 \mathrm{~b}$ & $4.4 \mathrm{~b}$ & $\mathrm{~ns}$ & \\
\hline $6-8$ & $5.6 \mathrm{~b}$ & $5.3 \mathrm{~b}$ & $4.7 \mathrm{~b}$ & $4.8 \mathrm{~b}$ & $\mathrm{~ns}$ & \\
\hline $8-10$ & $5.5 \mathrm{~b}$ & $5.6 \mathrm{~b}$ & $4.7 \mathrm{~b}$ & $4.5 \mathrm{~b}$ & $\mathrm{~ns}$ & \\
\hline $10-12$ & $5.9 \mathrm{~b}$ & $5.6 \mathrm{~b}$ & $4.7 \mathrm{~b}$ & $4.6 \mathrm{~b}$ & $\mathrm{~ns}$ & \\
\hline $12-14$ & $5.5 \mathrm{~b}$ & $5.4 \mathrm{~b}$ & $5.0 \mathrm{~b}$ & $4.8 \mathrm{~b}$ & $\mathrm{~ns}$ & \\
\hline $14-16$ & $5.6 \mathrm{~b}$ & $5.9 \mathrm{~b}$ & $5.2 \mathrm{~b}$ & $4.8 \mathrm{~b}$ & $\mathrm{~ns}$ & \\
\hline $16-18$ & $6.1 \mathrm{~b}$ & $5.9 \mathrm{~b}$ & $4.8 \mathrm{~b}$ & $4.9 \mathrm{~b}$ & $\mathrm{~ns}$ & \\
\hline $18-20$ & $6.2 \mathrm{~b}$ & $6.3 \mathrm{~b}$ & $4.7 \mathrm{~b}$ & $4.9 \mathrm{~b}$ & $\mathrm{~ns}$ & \\
\hline $20-25$ & $6.9 \mathrm{~b}$ & $6.4 \mathrm{~b}$ & $5.1 \mathrm{~b}$ & $5.1 \mathrm{~b}$ & $\mathrm{~ns}$ & \\
\hline $25-30$ & $7.8 \mathrm{a}$ & $6.3 \mathrm{~b}$ & $5.6 \mathrm{~b}$ & $5.3 \mathrm{~b}$ & $\mathrm{~ns}$ & \\
\hline $30-35$ & $7.8 \mathrm{a}$ & $7.1 \mathrm{a}$ & $6.1 \mathrm{a}$ & $6.0 \mathrm{a}$ & $\mathrm{ns}$ & \\
\hline $35-40$ & $8.4 \mathrm{a}$ & $7.0 \mathrm{a}$ & $6.9 \mathrm{a}$ & $6.1 \mathrm{a}$ & $\mathrm{ns}$ & \\
\hline $40-50$ & $8.5 \mathrm{a}$ & $7.6 \mathrm{a}$ & $7.3 \mathrm{a}$ & $7.0 \mathrm{a}$ & $\mathrm{ns}$ & \\
\hline $50-60$ & $8.8 \mathrm{a}$ & $8.3 \mathrm{a}$ & $7.4 \mathrm{a}$ & $7.1 \mathrm{a}$ & $\mathrm{ns}$ & \\
\hline CV (\%) & 14.2 & 17.0 & 13.1 & 16.6 & & \\
\hline
\end{tabular}

(1) Averages followed by the same letter in columns did not differ from each other by the Scott-Knott test ( $\alpha=0.05)$; ns: not significant at $5 \% ;{ }^{*}$ : significant at $5 \%$. 
5.0 and 6.0 the waste application may have no effect. An example is the report of Scherer et al. (2007), who conducted two experiments with pig slurry applications of 0,40 and $115 \mathrm{~m}^{3} \mathrm{ha}^{-1}$ year $\mathrm{r}^{-1}$, one that lasted four years in Chapecó (SC), and the other three years in Guatambu (SC). Both experiments were carried out on a Typic Hapludox, with water $\mathrm{pH}$ values (at the beginning of the experiment) of 5.4 and 5.2 , respectively. On the other hand, manure application to soils with $\mathrm{pH}$ close to neutrality, can induce a lowering of the $\mathrm{pH}$ values. This was observed in the United States by Adeli et al. (2008), in a study on pig slurry application to a soil with initial $\mathrm{pH}$ of 6.9 where, after applying the waste for 15 years, the soil $\mathrm{pH}$ reached 5.9 in the $0-15 \mathrm{~cm}$ layer. According to these authors, this decrease of soil $\mathrm{pH}$ values over time is a consequence of the base leaching from the soil profile. In an opposite situation, Assmann et al. (2007) observed that the $\mathrm{pH}$ of an Oxisol with initial $\mathrm{pH}$ of 4.52 , in the layer $0-20 \mathrm{~cm}$, under two applications of $80 \mathrm{~m}^{3} \mathrm{ha}^{-1}$ of pig slurry within 156 days, increased to 4.79. According to these studies, successive manure applications tends to induce an equilibrium in the soil $\mathrm{pH}$, within a range of approximately 5.0-6.0.

Although manure application induced no significant alterations, the potential acidity tended to decrease with increasing pig slurry rates (Table 2). This indicates that the period of applications was not long enough to manifest significant results regarding this property. However, continued applications of manure over longer periods can significantly reduce the values of potential acidity.

Al saturation was reduced by the pig slurry applications to a depth of $20 \mathrm{~cm}$ (Table 3). These results are relevant, because the manure application reduced $\mathrm{Al}$ saturation to values below $10 \%$, indicated by the CQFSRS/SC (2004) as non-limiting for notillage systems. Additionally, this reduction reached

Table 3. Values of $\mathrm{Al}$ saturation and base saturation in soil layers after 19 pig slurry applications at different rates

\begin{tabular}{|c|c|c|c|c|c|c|c|}
\hline \multirow{2}{*}{ Layer } & \multicolumn{4}{|c|}{ Rate of pig slurry $\left(\mathrm{m}^{3} \mathrm{ha}^{-1}\right)$} & \multirow{2}{*}{\multicolumn{2}{|c|}{$\begin{array}{c}\text { Regression } \\
\text { Equation }\end{array}$}} & \multirow{2}{*}{$\mathbf{R}^{2}$} \\
\hline & $\mathbf{0}$ & 20 & 40 & 80 & & & \\
\hline $\mathrm{cm}$ & \multicolumn{4}{|c|}{ Al Saturation (\%) } & & & \\
\hline $0-2$ & $2.3 \mathrm{e}^{(1)}$ & $1.4 \mathrm{e}^{(1)}$ & $1.0 \mathrm{e}^{(1)}$ & $1.0 \mathrm{~d}^{(1)}$ & $\hat{y}=1.902$ & $-0.015 x$ & 0.67 * \\
\hline $2-4$ & $5.5 \mathrm{e}$ & $2.1 \mathrm{e}$ & $2.4 \mathrm{e}$ & $2.2 \mathrm{~d}$ & $\hat{y}=4.183$ & $-0.032 x$ & $0.43^{*}$ \\
\hline $4-6$ & $9.8 \mathrm{~d}$ & $5.2 \mathrm{e}$ & $4.3 \mathrm{e}$ & $2.4 \mathrm{~d}$ & $\hat{y}=8.314$ & $-0.083 x$ & $0.82^{*}$ \\
\hline $6-8$ & $10.6 \mathrm{~d}$ & $6.3 \mathrm{~d}$ & $5.0 \mathrm{e}$ & $2.2 \mathrm{~d}$ & $\hat{\mathrm{y}}=9.474$ & $-0.098 x$ & 0.91 * \\
\hline $8-10$ & $11.2 \mathrm{~d}$ & $8.2 \mathrm{~d}$ & $5.2 \mathrm{e}$ & $2.2 \mathrm{~d}$ & $\hat{y}=10.616$ & $-0.111 x$ & 0.96 * \\
\hline $10-12$ & $12.1 \mathrm{~d}$ & $9.7 \mathrm{~d}$ & $5.9 \mathrm{e}$ & $2.8 \mathrm{~d}$ & $\hat{\mathrm{y}}=11.743$ & $-0.118 x$ & 0.96 * \\
\hline $12-14$ & $13.6 \mathrm{c}$ & $8.4 \mathrm{~d}$ & $6.4 \mathrm{e}$ & $3.6 \mathrm{~d}$ & $\hat{y}=12.085$ & $-0.117 x$ & 0.89 * \\
\hline $14-16$ & $16.8 \mathrm{c}$ & $9.4 \mathrm{~d}$ & $6.4 \mathrm{e}$ & $3.7 \mathrm{~d}$ & $\hat{y}=14.342$ & $-0.151 x$ & $0.83^{*}$ \\
\hline $16-18$ & $17.8 \mathrm{c}$ & $9.0 \mathrm{~d}$ & $5.9 \mathrm{e}$ & $3.8 \mathrm{~d}$ & $\hat{\mathrm{y}}=14.678$ & $-0.159 x$ & $0.78^{*}$ \\
\hline $18-20$ & $18.4 \mathrm{c}$ & $11.1 \mathrm{~d}$ & $7.0 \mathrm{e}$ & $4.8 \mathrm{~d}$ & $\hat{y}=15.898$ & $-0.160 x$ & 0.84 * \\
\hline $20-25$ & $18.4 \mathrm{c}$ & $10.8 \mathrm{~d}$ & $7.3 \mathrm{e}$ & $9.8 \mathrm{c}$ & $\mathrm{n}$ & $\mathrm{s}$ & \\
\hline $25-30$ & $18.6 \mathrm{c}$ & $17.4 \mathrm{c}$ & $12.0 \mathrm{~d}$ & $13.7 \mathrm{c}$ & $\mathrm{n}$ & s & \\
\hline $30-35$ & $27.2 \mathrm{~b}$ & $19.6 \mathrm{c}$ & $18.4 \mathrm{c}$ & $19.4 \mathrm{~b}$ & $\mathrm{n}$ & s & \\
\hline $35-40$ & $34.1 \mathrm{~b}$ & $29.1 \mathrm{~b}$ & $24.0 \mathrm{~b}$ & $25.8 \mathrm{a}$ & $\mathrm{n}$ & $\mathrm{s}$ & \\
\hline $40-50$ & $45.4 \mathrm{a}$ & $33.7 \mathrm{~b}$ & $35.3 \mathrm{a}$ & $28.6 \mathrm{a}$ & $\mathrm{n}$ & $\mathrm{s}$ & \\
\hline $50-60$ & 48.9 a & $39.5 \mathrm{a}$ & $39.4 \mathrm{a}$ & $29.7 \mathrm{a}$ & $\mathrm{n}$ & $\mathrm{s}$ & \\
\hline \multirow[t]{2}{*}{$\mathrm{CV}(\%)$} & 22.2 & 28.6 & 26.8 & 31.3 & & & \\
\hline & \multicolumn{4}{|c|}{ Base saturation (\%) } & & & \\
\hline $0-2$ & $52.0 \mathrm{a}^{(1)}$ & $59.8 \mathrm{a}^{(1)}$ & $63.8 \mathrm{a}^{(1)}$ & $68.5 \mathrm{a}^{(1)}$ & $\hat{y}=54.175$ & $+0.196 x$ & 0.91 * \\
\hline $2-4$ & $42.9 \mathrm{~b}$ & $52.0 \mathrm{~b}$ & $58.3 \mathrm{a}$ & $62.2 \mathrm{a}$ & $\hat{\mathrm{y}}=45.753$ & $+0.231 \mathrm{x}$ & 0.88 * \\
\hline $4-6$ & $40.1 \mathrm{~b}$ & $46.6 \mathrm{c}$ & $54.3 \mathrm{~b}$ & $59.9 \mathrm{a}$ & $\hat{\mathrm{y}}=41.579$ & $+0.247 x$ & 0.94 * \\
\hline $6-8$ & $40.7 \mathrm{~b}$ & $44.8 \mathrm{c}$ & $53.0 \mathrm{~b}$ & $57.6 \mathrm{~b}$ & $\hat{\mathrm{y}}=41.382$ & $+0.218 x$ & 0.94 * \\
\hline $8-10$ & $39.2 \mathrm{~b}$ & $44.1 \mathrm{c}$ & $52.3 \mathrm{~b}$ & $56.6 \mathrm{~b}$ & $\hat{\mathrm{y}}=40.310$ & $+0.221 x$ & 0.93 * \\
\hline $10-12$ & $39.4 \mathrm{~b}$ & $43.5 \mathrm{c}$ & $51.0 \mathrm{~b}$ & $55.4 \mathrm{~b}$ & $\hat{\mathrm{y}}=40.117$ & $+0.205 x$ & 0.94 * \\
\hline $12-14$ & $40.6 \mathrm{~b}$ & $43.7 \mathrm{c}$ & $50.6 \mathrm{~b}$ & $54.4 \mathrm{~b}$ & $\mathrm{n}$ & s & \\
\hline $14-16$ & $39.6 \mathrm{~b}$ & $40.7 \mathrm{c}$ & $50.3 \mathrm{~b}$ & $54.0 \mathrm{~b}$ & $\mathrm{n}$ & $\mathrm{s}$ & \\
\hline $16-18$ & $39.1 \mathrm{~b}$ & $39.4 \mathrm{c}$ & $50.1 \mathrm{~b}$ & $52.9 \mathrm{~b}$ & $\mathrm{n}$ & & \\
\hline $18-20$ & $38.9 \mathrm{~b}$ & $39.3 \mathrm{c}$ & $50.3 \mathrm{~b}$ & $50.6 \mathrm{~b}$ & $\mathrm{n}$ & $\mathrm{s}$ & \\
\hline $20-25$ & $35.6 \mathrm{~b}$ & $39.4 \mathrm{c}$ & $47.3 \mathrm{c}$ & $44.7 \mathrm{~b}$ & $\mathrm{n}$ & $\mathrm{s}$ & \\
\hline $25-30$ & $31.9 \mathrm{c}$ & $36.7 \mathrm{~d}$ & $42.5 \mathrm{c}$ & $40.1 \mathrm{c}$ & $\mathrm{n}$ & $\mathrm{s}$ & \\
\hline $30-35$ & $28.9 \mathrm{c}$ & $31.9 \mathrm{~d}$ & $37.5 \mathrm{~d}$ & $36.2 \mathrm{c}$ & $\mathrm{n}$ & $\mathrm{s}$ & \\
\hline $35-40$ & $25.3 \mathrm{c}$ & $27.2 \mathrm{~d}$ & $32.8 \mathrm{~d}$ & $33.9 \mathrm{~d}$ & $\mathrm{n}$ & $\mathrm{s}$ & \\
\hline $40-50$ & $20.2 \mathrm{~d}$ & $25.2 \mathrm{e}$ & $27.7 \mathrm{e}$ & $30.3 \mathrm{~d}$ & $\mathrm{n}$ & $\mathrm{s}$ & \\
\hline $50-60$ & $19.3 \mathrm{~d}$ & $23.1 \mathrm{e}$ & $24.9 \mathrm{e}$ & $30.1 \mathrm{~d}$ & $\mathrm{n}$ & & \\
\hline CV (\%) & 10.0 & 10.5 & 6.9 & 9.0 & & & \\
\hline
\end{tabular}

(1) Averages followed by the same letter in columns did not differ from each other by the Scott-Knott test $(\alpha=0.05)$; ns: not significant at $5 \%$; *: significant at $5 \%$. 
deeper than $10 \mathrm{~cm}$, which is the depth at which soil is sampled for analyses and recommendation of manure and liming requirements in the no-tillage system. These aspects have a positive impact on plant growth and represent an improvement of the environment for root growth in deeper layers. The reduction in $\mathrm{Al}$ saturation motivated by the use of manure was probably related to the complexation of exchangeable $\mathrm{Al}$, especially the fulvic and humic acid fractions of SOM, which are very reactive and increase significantly in the soil when high $\mathrm{C}$ quantities are applied. This explanation was proposed by Ceretta et al. (2003) who observed a decrease in $\mathrm{Al}$ saturation in the Hapludult under natural pasture and treated with pig slurry at rates of 0,20 and $40 \mathrm{~m}^{3} \mathrm{ha}^{-1}$ for four years. The reduction in $\mathrm{Al}$ saturation can also be explained by the rise in soil $\mathrm{pH}$ values (Table 2 ), once the increase in the concentrations of $\mathrm{OH}^{-}$groups causes $\mathrm{Al}^{3+}$ hydrolysis in the soil and, consequently, reduces $\mathrm{Al}$ saturation. Reduction in the $\mathrm{Al}$ levels were also observed by Assmann et al. (2007) in Oxisol after two pig slurry applications of $0,20,40$ and $80 \mathrm{~m}^{3} \mathrm{ha}^{-1}$.

The successive pig slurry applications increased base saturation to a depth of $12 \mathrm{~cm}$ (Table 3). For notillage soils, a base saturation of $\geq 65 \%$ is recommended by the CQFSRS/SC (2004), which was only reached in layer levels of $0-2 \mathrm{~cm}$, where $80 \mathrm{~m}^{3} \mathrm{ha}^{-1}$ of manure was applied (68.5\%). These results can be attributed to the increase in SOM levels, which contributes to the adsorption of soil cations, as well as to the addition of $\mathrm{Ca}$ and $\mathrm{Mg}$ with manure applications.

The $\mathrm{Ca}$ and $\mathrm{Mg}$ levels increased, respectively, to a depth of 16 and $20 \mathrm{~cm}$ (Table 4). For $\mathrm{Ca}$, the increments to $16 \mathrm{~cm}$ were on average 11,36 and $48 \%$, at rates of 20,40 and $80 \mathrm{~m}^{3} \mathrm{ha}^{-1}$, respectively, compared to the treatment without manure application. For $\mathrm{Mg}$, the increments down to $20 \mathrm{~cm}$ were on average 10,32 and $58 \%$ at rates of 20,40

Table 4. Values of exchangeable Ca and Mg in soil layers after 19 pig slurry applications at different rates

\begin{tabular}{|c|c|c|c|c|c|c|}
\hline \multirow{2}{*}{ Layer } & \multicolumn{4}{|c|}{ Rate of pig slurry $\left(\mathrm{m}^{3} \mathrm{ha}^{-1}\right)$} & \multirow{2}{*}{$\begin{array}{c}\text { Regression } \\
\text { Equation }\end{array}$} & \multirow{2}{*}{$\mathbf{R}^{2}$} \\
\hline & 0 & 20 & 40 & 80 & & \\
\hline $\mathrm{cm}$ & \multicolumn{5}{|c|}{ Exchangeable $\mathrm{Ca}\left(\mathrm{cmol}_{\mathrm{c}} \mathrm{dm}^{-3}\right)$} & \\
\hline $0-2$ & $2.6 \mathrm{a}^{(1)}$ & $1.4 \mathrm{e}^{(1)}$ & $3.6 \mathrm{a}^{(1)}$ & $4.3 \mathrm{a}^{(1)}$ & $\hat{y}=2.578+0.022 x$ & 0.98 * \\
\hline $2-4$ & $2.1 \mathrm{~b}$ & $2.1 \mathrm{e}$ & $3.3 \mathrm{~b}$ & $3.6 \mathrm{~b}$ & $\hat{y}=2.268+0.018 x$ & 0.88 * \\
\hline $4-6$ & $2.0 \mathrm{~b}$ & $5.2 \mathrm{e}$ & $3.0 \mathrm{c}$ & $3.3 \mathrm{c}$ & $\hat{\mathrm{y}}=2.058+0.017 \mathrm{x}$ & 0.93 * \\
\hline $6-8$ & $2.1 \mathrm{~b}$ & $6.3 \mathrm{~d}$ & $2.8 \mathrm{c}$ & $3.1 \mathrm{c}$ & $\hat{\mathrm{y}}=2.102+0.013 \mathrm{x}$ & 0.93 * \\
\hline $8-10$ & $2.1 \mathrm{~b}$ & $8.2 \mathrm{~d}$ & $2.6 \mathrm{c}$ & $2.9 \mathrm{c}$ & $\hat{y}=2.116+0.010 x$ & 0.97 * \\
\hline $10-12$ & $2.1 \mathrm{~b}$ & $9.7 \mathrm{~d}$ & $2.6 \mathrm{c}$ & $2.8 \mathrm{~d}$ & $\hat{y}=2.136+0.009 x$ & 0.87 * \\
\hline $12-14$ & $2.1 \mathrm{~b}$ & $8.4 \mathrm{~d}$ & $2.7 \mathrm{c}$ & $2.7 \mathrm{~d}$ & $\hat{\mathrm{y}}=2.128+0.009 \mathrm{x}$ & 0.77 * \\
\hline $14-16$ & $2.0 \mathrm{~b}$ & $9.4 \mathrm{~d}$ & $2.7 \mathrm{c}$ & $2.7 \mathrm{~d}$ & $\hat{\mathrm{y}}=2.080+0.009 \mathrm{x}$ & 0.73 * \\
\hline $16-18$ & $2.1 \mathrm{~b}$ & $9.0 \mathrm{~d}$ & $2.7 \mathrm{c}$ & $2.5 \mathrm{~d}$ & ns & \\
\hline $18-20$ & $2.0 \mathrm{~b}$ & $11.1 \mathrm{~d}$ & $2.7 \mathrm{c}$ & $2.6 \mathrm{~d}$ & $\mathrm{~ns}$ & \\
\hline $20-25$ & $2.0 \mathrm{~b}$ & $10.8 \mathrm{~d}$ & $2.6 \mathrm{c}$ & $2.2 \mathrm{e}$ & $\mathrm{ns}$ & \\
\hline $25-30$ & $1.6 \mathrm{c}$ & $17.4 \mathrm{c}$ & $2.3 \mathrm{~d}$ & $2.0 \mathrm{e}$ & $\mathrm{ns}$ & \\
\hline $30-35$ & $1.4 \mathrm{c}$ & $19.6 \mathrm{c}$ & $2.0 \mathrm{e}$ & $1.9 \mathrm{e}$ & $\mathrm{ns}$ & \\
\hline $35-40$ & $1.2 \mathrm{~d}$ & $29.1 \mathrm{~b}$ & $1.7 \mathrm{e}$ & $1.6 \mathrm{f}$ & $\mathrm{ns}$ & \\
\hline $40-50$ & $1.0 \mathrm{~d}$ & $33.7 \mathrm{~b}$ & $1.4 \mathrm{f}$ & $1.5 \mathrm{f}$ & $\mathrm{ns}$ & \\
\hline $50-60$ & $0.9 \mathrm{~d}$ & $39.5 \mathrm{a}$ & $1.2 \mathrm{f}$ & $1.6 \mathrm{f}$ & $\mathrm{ns}$ & \\
\hline \multirow[t]{2}{*}{$\mathrm{CV}(\%)$} & 11.8 & 28.6 & 6.0 & 7.7 & & \\
\hline & \multicolumn{4}{|c|}{ Exchangeable $\mathrm{Mg}\left(\mathrm{cmol}_{\mathrm{c}} \mathrm{dm}^{-3}\right)$} & & \\
\hline $0-2$ & $2.4 \mathrm{a}^{(1)}$ & $59.8 \mathrm{a}^{(1)}$ & $2.9 \mathrm{a}^{(1)}$ & $3.5 \mathrm{a}^{(1)}$ & $\hat{\mathrm{y}}=2.348+0.014 \mathrm{x}$ & 0.99 * \\
\hline $2-4$ & $1.9 \mathrm{~b}$ & $52.0 \mathrm{~b}$ & $2.7 \mathrm{a}$ & $3.2 \mathrm{a}$ & $\hat{\mathrm{y}}=2.006+0.016 \mathrm{x}$ & 0.98 * \\
\hline $4-6$ & $1.7 \mathrm{~b}$ & $46.6 \mathrm{c}$ & $2.5 \mathrm{~b}$ & $3.1 \mathrm{a}$ & $\hat{\mathrm{y}}=1.752+0.017 \mathrm{x}$ & 0.99 * \\
\hline $6-8$ & $1.7 \mathrm{~b}$ & $44.8 \mathrm{c}$ & $2.4 \mathrm{~b}$ & 2.7 & $\hat{\mathrm{y}}=1.752+0.012 \mathrm{x}$ & 0.96 * \\
\hline $8-10$ & $1.8 \mathrm{~b}$ & $44.1 \mathrm{c}$ & $2.4 \mathrm{~b}$ & $2.8 \mathrm{~b}$ & $\hat{\mathrm{y}}=1.740+0.013 \mathrm{x}$ & 0.98 * \\
\hline $10-12$ & $1.7 \mathrm{~b}$ & $43.5 \mathrm{c}$ & $2.2 \mathrm{c}$ & $2.8 \mathrm{~b}$ & $\hat{\mathrm{y}}=1.622+0.014 \mathrm{x}$ & 0.99 * \\
\hline $12-14$ & $1.6 \mathrm{~b}$ & $43.7 \mathrm{c}$ & $2.1 \mathrm{c}$ & $2.6 \mathrm{~b}$ & $\hat{\mathrm{y}}=1.550+0.013 \mathrm{x}$ & 0.98 * \\
\hline $14-16$ & $1.6 \mathrm{~b}$ & $40.7 \mathrm{c}$ & $2.1 \mathrm{c}$ & $2.5 \mathrm{~b}$ & $\hat{\mathrm{y}}=1.552+0.012 \mathrm{x}$ & 0.98 * \\
\hline $16-18$ & $1.6 \mathrm{~b}$ & $39.4 \mathrm{c}$ & $2.0 \mathrm{c}$ & $2.4 \mathrm{~b}$ & $\hat{\mathrm{y}}=1.578+0.010 \mathrm{x}$ & 0.94 * \\
\hline $18-20$ & $1.6 \mathrm{~b}$ & $39.3 \mathrm{c}$ & $2.0 \mathrm{~d}$ & $2.3 \mathrm{~b}$ & $\hat{\mathrm{y}}=1.542+0.009 \mathrm{x}$ & 0.95 * \\
\hline $20-25$ & $1.6 \mathrm{~b}$ & $39.4 \mathrm{c}$ & $1.9 \mathrm{~d}$ & $2.0 \mathrm{c}$ & ns & \\
\hline $25-30$ & $1.6 \mathrm{~b}$ & $36.7 \mathrm{~d}$ & $1.8 \mathrm{~d}$ & $1.7 \mathrm{c}$ & $\mathrm{ns}$ & \\
\hline $30-35$ & $1.4 \mathrm{c}$ & $31.9 \mathrm{~d}$ & $1.6 \mathrm{e}$ & $1.6 \mathrm{~d}$ & ns & \\
\hline $35-40$ & $1.3 \mathrm{c}$ & $27.2 \mathrm{~d}$ & $1.5 \mathrm{e}$ & $1.5 \mathrm{~d}$ & $\mathrm{~ns}$ & \\
\hline $40-50$ & $1.1 \mathrm{~d}$ & $25.2 \mathrm{e}$ & $1.3 \mathrm{f}$ & $1.3 \mathrm{~d}$ & $\mathrm{~ns}$ & \\
\hline $50-60$ & $1.0 \mathrm{~d}$ & $23.1 \mathrm{e}$ & $1.2 \mathrm{f}$ & $1.3 \mathrm{~d}$ & $\mathrm{~ns}$ & \\
\hline $\mathrm{CV}(\%)$ & 12.4 & 10.5 & 5.4 & 11.4 & & \\
\hline
\end{tabular}

(1) Averages followed by the same letter in columns did not differ from each other by the Scott-Knott test $(\alpha=0.05)$; ns: not significant at $5 \%$; *: significant at $5 \%$. 
and $80 \mathrm{~m}^{3} \mathrm{ha}^{-1}$, respectively, according to the treatment without manure application. This can be justified, mainly, by the quantities of $\mathrm{Ca}$ and $\mathrm{Mg}$ contained in the 19 manure applications over 100 months (i.e., 327, 654 and $1307 \mathrm{~kg} \mathrm{ha}^{-1} \mathrm{Ca}$ and 378, 756 and $1514 \mathrm{~kg} \mathrm{ha}^{-1} \mathrm{Mg}$ at 20, 40 and $80 \mathrm{~m}^{3} \mathrm{ha}^{-1}$ residues, respectively). The percolation of watersoluble organic compounds and the maintenance of biopores, channels formed by the growth and deterioration of crop roots in no-tillage soils were factors mentioned by Kaminski et al. (2005) to explain the accumulation of $\mathrm{Ca}$ and $\mathrm{Mg}$ in the deeper soil layers. Ceretta et al. (2003) found an increase of 49 and $40 \%$ in the Ca levels in the layers 0-2.5 and 2.5$5.0 \mathrm{~cm}$, respectively, after application of 28 rates of $40 \mathrm{~m}^{3} \mathrm{ha}^{-1}$ of pig slurry to natural pasture in four years. According to these authors, the increase in $\mathrm{Ca}$ soil percentage is relatively small and can be explained by the low $\mathrm{Ca}$ concentration in the manure, the possibility of mineral compound formation with phosphates or the higher adsorption of Ca in SOM, which complicates the estimation by extractor $\mathrm{KCl}$ $1 \mathrm{~mol} \mathrm{~L}^{-1}$. Regarding $\mathrm{Mg}$, the authors found increments in the nutrient levels to a depth of $20 \mathrm{~cm}$, when $40 \mathrm{~m}^{3} \mathrm{ha}^{-1}$ of pig slurry was applied.

Soil organic matter increased on average 30,36 and $74 \%$, to a depth of $60 \mathrm{~cm}$, by the applications of 20, 40 and $80 \mathrm{~m}^{3} \mathrm{ha}^{-1}$ of pig slurry (Table 5). This may result from the migration of organic compounds of low molecular weight down the soil profile, provoked by saturation of the functional groups of surface soil due to the addition of high quantities of organic compounds in manure. The levels of soil clay, silt and sand of 170,300 and $530 \mathrm{~g} \mathrm{~kg}^{-1}$, respectively, is also an aspect that favored the downward movement of organic compounds that explain this increment down to $60 \mathrm{~cm}$. Notwithstanding, the highest SOM increments occurred at manure rates of $80 \mathrm{~m}^{3} \mathrm{ha}^{-1}$, especially in the $0-2 \mathrm{~cm}$ soil layer, where levels reached $62.5 \mathrm{~g} \mathrm{~kg}^{-1}$, while in the area without manure

Table 5. Values of organic matter and CEC potential in soil layers after nineteen pig slurry applications at different rates

\begin{tabular}{|c|c|c|c|c|c|c|}
\hline \multirow{2}{*}{ Layer } & \multicolumn{4}{|c|}{ Rate of pig slurry $\left(\mathrm{m}^{3} \mathrm{ha}^{-1}\right)$} & \multirow{2}{*}{$\begin{array}{c}\text { Regression } \\
\text { Equation }\end{array}$} & \multirow{2}{*}{$\mathbf{R}^{2}$} \\
\hline & $\mathbf{0}$ & 20 & 40 & 80 & & \\
\hline $\mathrm{cm}$ & \multicolumn{5}{|c|}{ Organic Matter ( $\mathrm{g} \mathrm{kg}^{-1}$ ) } & \\
\hline $0-2$ & $27.2 \mathrm{a}^{(1)}$ & $39.0 \mathrm{a}^{(1)}$ & $42.5 \mathrm{a}^{(1)}$ & $62.5 \mathrm{a}^{(1)}$ & $\hat{y}=27.866+0.426 x$ & $0.98^{*}$ \\
\hline $2-4$ & $20.9 \mathrm{~b}$ & $29.5 \mathrm{~b}$ & $32.8 \mathrm{~b}$ & $42.1 \mathrm{~b}$ & $\hat{\mathrm{y}}=22.490+0.252 \mathrm{x}$ & 0.97 * \\
\hline $4-6$ & $17.0 \mathrm{c}$ & $23.8 \mathrm{c}$ & $25.4 \mathrm{c}$ & $32.1 \mathrm{c}$ & $\hat{y}=18.368+0.177 x$ & 0.95 * \\
\hline $6-8$ & $15.6 \mathrm{c}$ & $21.3 \mathrm{~d}$ & $20.8 \mathrm{~d}$ & $27.4 \mathrm{~d}$ & $\hat{y}=16.590+0.135 x$ & 0.91 * \\
\hline $8-10$ & $14.2 \mathrm{~d}$ & $19.4 \mathrm{e}$ & $18.4 \mathrm{e}$ & $24.0 \mathrm{e}$ & $\hat{\mathrm{y}}=15.150+0.110 \mathrm{x}$ & $0.86^{*}$ \\
\hline $10-12$ & $13.5 \mathrm{~d}$ & $18.0 \mathrm{e}$ & $18.2 \mathrm{e}$ & $22.2 \mathrm{e}$ & $\hat{y}=14.486+0.099 x$ & 0.91 * \\
\hline $12-14$ & $13.8 \mathrm{~d}$ & $18.0 \mathrm{e}$ & $17.5 \mathrm{e}$ & $20.0 \mathrm{f}$ & $\hat{\mathrm{y}}=15.006+0.066 \mathrm{x}$ & $0.78^{*}$ \\
\hline $14-16$ & $13.0 \mathrm{~d}$ & $17.4 \mathrm{e}$ & $17.5 \mathrm{e}$ & $20.2 \mathrm{f}$ & $\hat{\mathrm{y}}=14.218+0.080 \mathrm{x}$ & $0.84^{*}$ \\
\hline $16-18$ & $14.1 \mathrm{~d}$ & $17.4 \mathrm{e}$ & $17.9 \mathrm{e}$ & $20.8 \mathrm{f}$ & $\hat{\mathrm{y}}=14.818+0.077 \mathrm{x}$ & $0.93^{*}$ \\
\hline $18-20$ & $13.0 \mathrm{~d}$ & $17.7 \mathrm{e}$ & $17.8 \mathrm{e}$ & $23.4 \mathrm{e}$ & $\hat{y}=13.770+0.120 x$ & 0.94 * \\
\hline $20-25$ & $12.4 \mathrm{~d}$ & $16.7 \mathrm{e}$ & $18.3 \mathrm{e}$ & $24.1 \mathrm{e}$ & $\hat{\mathrm{y}}=12.967+0.140 \mathrm{x}$ & 0.98 * \\
\hline $25-30$ & $14.7 \mathrm{~d}$ & $19.2 \mathrm{e}$ & $20.1 \mathrm{~d}$ & $23.7 \mathrm{e}$ & $\hat{\mathrm{y}}=15.749+0.105 x$ & $0.93^{*}$ \\
\hline $30-35$ & $17.1 \mathrm{c}$ & $17.6 \mathrm{e}$ & $20.0 \mathrm{~d}$ & $23.6 \mathrm{e}$ & $\hat{\mathrm{y}}=16.571+0.086 \mathrm{x}$ & $0.97^{*}$ \\
\hline $35-40$ & $15.8 \mathrm{c}$ & $17.0 \mathrm{e}$ & $19.1 \mathrm{e}$ & $23.1 \mathrm{e}$ & $\hat{\mathrm{y}}=15.448+0.094 \mathrm{x}$ & 0.99 * \\
\hline $40-50$ & $14.0 \mathrm{~d}$ & $16.4 \mathrm{e}$ & $16.1 \mathrm{f}$ & $21.3 \mathrm{f}$ & $\hat{y}=13.898+0.087 x$ & $0.92 *$ \\
\hline $50-60$ & $11.1 \mathrm{~d}$ & $12.4 \mathrm{f}$ & $14.5 \mathrm{~g}$ & $19.0 \mathrm{f}$ & $\hat{\mathrm{y}}=10.707+0.101 \mathrm{x}$ & 0.99 * \\
\hline \multirow[t]{2}{*}{$\mathrm{CV}(\%)$} & 9.0 & 6.5 & $4.3^{\circ}$ & 6.0 & & \\
\hline & \multicolumn{4}{|c|}{$\mathrm{CEC}_{\mathrm{pH} 7}\left(\mathrm{cmol}_{\mathrm{c}} \mathrm{dm}^{-3}\right)$} & & \\
\hline $0-2$ & $10.2^{\mathrm{ns}}$ & $9.9^{\mathrm{ns}}$ & $10.7^{\mathrm{ns}}$ & $12.2 \mathrm{a}^{(1)}$ & $\hat{y}=9.780+0.027 x$ & $0.84^{* *}$ \\
\hline $2-4$ & 9.7 & 10.0 & 10.7 & $11.4 \mathrm{a}$ & ns & 0.98 * \\
\hline $4-6$ & 9.5 & 9.8 & 10.2 & $11.1 \mathrm{a}$ & $\mathrm{ns}$ & 0.99 * \\
\hline $6-8$ & 9.6 & 9.6 & 9.9 & $10.8 \mathrm{a}$ & $\mathrm{ns}$ & $0.96^{*}$ \\
\hline $8-10$ & 10.0 & 9.9 & 9.7 & $10.4 \mathrm{~b}$ & $\mathrm{~ns}$ & 0.98 * \\
\hline $10-12$ & 9.7 & 9.8 & 9.6 & $10.3 \mathrm{~b}$ & $\mathrm{~ns}$ & 0.99 * \\
\hline $12-14$ & 9.2 & 9.5 & 10.0 & $10.3 \mathrm{~b}$ & $\mathrm{~ns}$ & 0.98 * \\
\hline $14-16$ & 9.3 & 9.9 & 10.1 & $10.1 \mathrm{~b}$ & $\mathrm{~ns}$ & 0.98 * \\
\hline $16-18$ & 9.8 & 9.6 & 9.6 & $10.0 \mathrm{~b}$ & $\mathrm{~ns}$ & 0.94 * \\
\hline $18-20$ & 9.9 & 10.0 & 9.4 & $9.9 \mathrm{~b}$ & $\mathrm{~ns}$ & 0.95 * \\
\hline $20-25$ & 10.5 & 10.1 & 9.7 & $9.4 \mathrm{~b}$ & $\mathrm{~ns}$ & \\
\hline $25-30$ & 11.1 & 9.7 & 9.7 & $9.1 \mathrm{~b}$ & $\mathrm{~ns}$ & \\
\hline $30-35$ & 10.6 & 10.2 & 9.7 & $9.5 \mathrm{~b}$ & $\mathrm{~ns}$ & \\
\hline $35-40$ & 11.0 & 9.8 & 10.2 & $9.3 \mathrm{~b}$ & $\mathrm{~ns}$ & \\
\hline $40-50$ & 10.5 & 10.1 & 10.0 & $9.8 \mathrm{~b}$ & $\mathrm{~ns}$ & \\
\hline $50-60$ & 10.8 & 10.7 & 9.8 & $10.0 \mathrm{~b}$ & $\mathrm{~ns}$ & \\
\hline CV (\%) & 8.6 & 11.1 & 7.0 & 8.5 & & \\
\hline
\end{tabular}

(1) Averages followed by the same letter in columns did not differ from each other by the Scott-Knott test $(\alpha=0.05)$; ns: not significant at $5 \%$; *: significant at $5 \%$. 
application, the level was $27.2 \mathrm{~g} \mathrm{~kg}^{-1}$. Surface effects can be justified, for instance, as stated by Falleiro et al. (2003), that in no-tillage systems, crop residues are maintained on the soil surface which slows down the mineralization rate, due to the reduced soil-residue interface, favoring SOM accumulation in the top soil layers.

The increase in SOM levels is caused by $\mathrm{C}$ addition, be it by manure or by crop dry matter input. To estimate the quantity of Organic Carbon (OC) added to soil in manure, a OC value of $28.5 \%$ in the dry matter of pig slurry was assumed. This percentage corresponds to studies conducted in the state of RS by Aita et al. (2006) and Giacomini \& Aita (2008), in which the average values were between 21.1 and $35.0 \%$ of OC in pig slurry dry matter. Thus, the soil OC after 19 waste applications was estimated at 4.93 , 9.85 , and $19.70 \mathrm{Mg} \mathrm{ha}^{-1}$ at pig slurry rates of 20,40 and $80 \mathrm{~m}^{3} \mathrm{ha}^{-1}$, respectively. Nevertheless, the highest contributions to the increase in SOM must have been the decomposition of crop residue in surface soil, once the accumulated production of dry matter by crops over 100 months was $57.9,92.1,109.2$, and $147.3 \mathrm{Mg} \mathrm{ha}^{-1}$ at pig slurry rates of $0,20,40$ and $80 \mathrm{~m}^{3} \mathrm{ha}^{-1}$, respectively.

One relevant aspect of SOM is its importance for colloidal properties, affecting the chemical properties in the soil such as CEC. However, despite the SOM increase to a depth of $60 \mathrm{~cm}$, the waste applications only increased CEC in the 0-2 cm layer (Table 5). This weak relation between SOM levels and soil CEC can be related to the low concentrations of phenolic and carboxylic groups in humic substances in manure, as observed by Hernandez et al. (2006). Moreover, these authors observed a reduction in phenolic and carboxylic groups in soils with manure application, and attributed this fact to the formation of organicmetallic complexes of functional groups with heavy metals, e.g., $\mathrm{Cu}$ and $\mathrm{Zn}$, applied with the residue. Summing up, the relevance of these results is in the fact that the pig slurry application raised the SOM levels and the quantity of matter added to soil by crops; this has very high positive impacts on nutrient cycling and the sustainability of the soil productive capacity.

For all variables analyzed in this study influenced by manure application, linear equations were generated, which indicated that the maximum effect of the pig slurry rates was not obtained. This indicated that, although the study was conducted for 8 years, with 19 pig slurry applications, these variables are still undergoing significant changes in different soil layers.

\section{CONCLUSIONS}

1. Successive applications of pig slurry decreased soil acidity to a depth of $8 \mathrm{~cm}$, without affecting potential acidity values.
2. The application of pig slurry induced accumulation of $\mathrm{Ca}^{2+}$ and $\mathrm{Mg}^{2+}$ in the surface soil layers, increasing base saturation and decreasing $\mathrm{Al}$ saturation.

3. Successive applications of pig slurry over eight years increased the levels of soil organic matter in deeper layers, but their effect on the potential CEC was restricted to the surface layer.

\section{LITERATURE CITED}

ADELI, A.; BOLSTER, C.H.; ROWE, D.E.; MCLAUGHLIN, M.R. \& BRINK, G.E. Effect of long-term swine effluent application on selected soil properties. Soil Sci., 173:223235,2008

AITA, C.; CHIAPINOTTO, I.C.; GIACOMINI, S.J.; HÜBNER, A.P. \& MARQUES, M.G. Decomposição de palha de aveia preta dejetos de suínos em solo sob plantio direto. R. Bras. Ci. Solo, 30:149-161, 2006.

ASSMANN, T.S.; ASSMANN, J.M.; CASSOL, L.C.; DIEHL, R.C.; MANTELI, C. \& MAGIERO, E.C. Desempenho da mistura forrageira de aveia-preta mais azevém e atributos químicos do solo em função da aplicação de esterco líquido de suínos. R. Bras. Ci. Solo, 31:1515-1523, 2007.

BASSO, C.J.; CERETTA, C.A.; DURIGON, R.; POLETTO, N. \& GIROTTO, E. Dejeto líquido de suínos: II - perdas de nitrogênio e fósforo por percolação no solo sob plantio direto. Ci. Rural, 35:1305-1312, 2005.

BERWANGER, A.L.; CERETTA, C.A. \& RHEINHEIMER, D.S. Alterações no teor de fósforo no solo com aplicação de dejetos líquidos de suínos. R. Bras. Ci. Solo, 32:2525-2532, 2008 .

BROWN, T.T.; KOENIG, R.T.; HARSH, J.B. \& ROSSI, R.E. Lime effects on soil acidity, crop yield, and aluminum chemistry in direct-seeded cropping systems. Soil Sci. Soc. Am. J., 72:634-640, 2008.

CERETTA, C.A.; BASSO, C.J.; VIEIRA, F.C.B.; HERBES, M.G.; MOREIRA, I.C.L. \& BERWANGER, A.L. Dejeto líquido de suínos: I-perdas de nitrogênio e fósforo na solução escoada na superfície do solo, sob plantio direto. Ci. Rural, 35:1296-1304, 2005.

CERETTA, C.A.; DURIGON, R.; BASSO, C.J.; BARCELLOS, L.A.R. \& VIEIRA, F.C.B. Características químicas de solo sob aplicação de esterco líquido de suínos em pastagem natural. Pesq. Agropec. Bras., 38:729-735, 2003.

CHANTIGNY, M.H.; ROCHETTE, P.; ANGERS, D.A.; MASSÉ, D. \& CÔTÉ, D. Ammonia volatilization and selected soil characteristics following application of anaerobically digested pig slurry. Soil Sci. Soc. Am. J., 68:306-312, 2004.

COMISSÃO DE QUÍMICA E FERTILIDADE DO SOLO CQFSRS-SC. Manual de adubação e calagem para os estados do Rio Grande do Sul e Santa Catarina. Porto Alegre, Sociedade Brasileira de Ciência do Solo/Núcleo Regional Sul, 2004. 400p. 
COMISSÃO DE FERTILIDADE DO SOLO - CFSRS/SC Recomendações de adubação e de calagem para os Estados do Rio Grande do Sul e Santa Catarina. Passo Fundo, CNPT/Embrapa, 1995. 224p.

EGHBALL, B. Soil properties as influenced by phosphorusand nitrogen-based manure and compost applications. Agron. J., 94:128-135, 2002.

EMPRESA BRASILEIRA DE PESQUISA AGROPECUÁRIA EMBRAPA. Manual de métodos de análises de solo. 2.ed. Rio de Janeiro, Ministério da Agricultura e do Abastecimento, 1997. 212p.

FALLEIRO, R.M.; SOUZA, C.M.; SILVA, C.S.W.; SEDIYAMA, C.S.; SILVA, A.A. \& FAGUNDES, J.L. Influência dos sistemas de preparo nas propriedades químicas e físicas do solo. R. Bras. Ci. Solo, 27:1097-1104, 2003.

GIACOMINI, S.J. \& AITA, C. Emissão de dióxido de carbono após aplicação de dejetos líquidos e cama sobreposta de suínos. Pesq. Agropec. Bras., 43:107-114, 2008.

GIROTTO, E. Cobre e zinco no solo sob uso intensivo de dejeto líquido de suínos. Santa Maria, Universidade Federal de Santa Maria, 2007. 121p. (Tese de Mestrado)

HERNÁNDEZ, D.; PLAZA, C.; SENESI, N. \& POLO, A. Detection of copper(II) and zinc(II) binding to humic acids from pig slurry and amended soils by ûuorescence spectroscopy. Environ. Poll., 143:212-220, 2006.

HUE, N.V. \& LICUDINE, D.L. Amelioration of subsoil acidity through surface application of organic manures. J. Environ. Qual., 28:623-632, 1999.

KAMINSKI, J.; RHEINHEIMER, D.S.; GATIBONI, L.C.; BRUNETTO, G. \& SILVA, L.S. Eficiência da calagem superficial e incorporada precedendo o sistema plantio direto em um Argissolo sob pastagem natural. R. Bras. Ci. Solo, 29:573-580, 2005.
McDOWELL, R.; SHARPLEY, A. \& FOLMAR, G. Phosphorus export from an agricultural watershed: Linking source and transport mechanisms. J. Environ. Qual., 30:1587$1595,2001$.

PAUL, J.W. \& BEAUCHAMP, E.G. Relationship between volatile fatty acids, total ammonia, and $\mathrm{pH}$ in manure slurries. Biol. Wastes, 29:313-318, 1989.

SCHERER, E.E.; BALDISSERA, I.T. \& NESI, C.N. Propriedades químicas de um Latossolo Vermelho sob plantio direto e adubação com esterco de suínos. R. Bras. Ci. Solo, 31:123-131, 2007.

SEGANFREDO, M.A. Os dejetos de suínos são um fertilizante ou um poluente do solo? Cader. Ci. Tecnol., 16:129-141, 1999.

SOIL SURVEY STAFF. Soil taxonomy. 2.ed. Washington, United States Department of Agriculture, 1999.

SORENSEN, P. Carbon mineralization, nitrogen immobilization and $\mathrm{pH}$ change in soil after adding volatile fatty acids. Europ. J. Soil Sci., 49:457-462, 1998.

TEDESCO, M.J.; GIANELLO, C.; BISSANI, C.A.; BOHNEN, H. \& VOLKWEISS, S.J. Análises de solo, planta e outros materiais. 2.ed. Porto Alegre, Universidade Federal do Rio Grande do Sul, 1995. 174p.

USDA - Soil Conservation Service. Soil survey investigations. Washington, 1972. 63p. (Report, 1)

WHALEN, J.K.; CHANG, C.; CLAYTON, G.W. \& CAREFOOT, J.P. Cattle manure amendments can increase the $\mathrm{pH}$ of acid soils. Soil Sci. Soc. Am. J., 64:962-966, 2000. 